Polymer Journal, Vol. 3, No. 3, pp 315-321 (1972)

\title{
Viscoelastic Properties of Concentrated Solutions of Poly(methyl methacrylate) in Diethyl Phthalate
}

\author{
Toshiro Masuda, Namihiro TodA*, Yoshio Aoto**, \\ and Shigeharu ONOGI \\ Department of Polymer Chemistry, Kyoto University, Kyoto, Japan.
}

(Received July 20, 1971)

\begin{abstract}
The viscoelastic properties of concentrated solutions of poly(methyl methacrylate) in diethyl phthalate were measured by means of a concentric cylinder type rheometer and capillary viscometer.

The Vogel parameters, $\alpha_{\mathrm{f}}$ and $T_{0}$ were calculated from the temperature dependence of viscosity and the shift factor $a_{\mathrm{T}}$ obtained from the time-temperature superposition. Moreover, the viscoelastic parameters characteristic of polymer liquids such as the zeroshear viscosity $\eta_{0}$, steady-state compliance $J_{e}{ }^{0}$, and entanglement compliance $J_{\mathrm{eN}}^{0}$ were evaluated from the frequency dependence of the storage shear modulus $G^{\prime}$ and the loss modulus $G^{\prime \prime}$.

With decreasing concentration, the Vogel temperature $T_{0}$ decreases at higher concentrations, but increases at lower concentrations after passing through a minimum at about $20 \mathrm{wt} \%$. The temperature coefficient of the fractional free volume, $\alpha_{\mathrm{f}}$, however, is almost constant at and above $20 \mathrm{wt} \%$, and then rapidly increases with decreasing concentration. This particular concentration of $20 \mathrm{wt} \%$ is very near the critical concentration of entanglement coupling.

$\eta_{0}$ is proportional to $w_{2}^{4.0}$, where $w_{2}$ is the weight fraction of polymer, in the isofree-volume state, and this exponent 4.0 is equal to that for molecular weight, which was obtained from the molecular weight dependence of $\eta_{0}$. Both $J_{\mathrm{e}^{0}}$ and $J_{\mathrm{eN}}^{0}$ are proportional to $w_{2}^{-2}$, and $J_{\mathrm{e}}^{0}$ is six times higher than $J_{\mathrm{eN}}^{0}$ over the entire range of concentration where $J_{\mathrm{eN}}^{0}$ can be evaluated.

KEY WORDS Concentrated Solution / Entanglement Coupling /

Poly(methyl methacrylate) / Steady-State Compliance / Viscoelasti-

city / The Vogel Parameters /
\end{abstract}

In our previous papers ${ }^{1-5}$ the viscoelastic properties of the binary blends of polymer in molten state have been measured extensively, and significant conclusions has been obtained about the dependence of the rheological properties of amorphous polymers upon molecular weight distribution. The main conclusions are summarized as follows: The Vogel temperature $T_{0}$ plotted against the weight fraction of the high molecular weight component, $w_{2}$, has a minimum at about $w_{2}=0.2$, while the temperature coefficient of fractional free volume, $\alpha_{\mathrm{f}}$, is almost constant over the entire range of $w_{2}$. The steady-

\footnotetext{
* Present address: Sumitomo Chemical Co., 40, Tsukahara, Takatsuki, Osaka, Japan.

** Present address: Kurayoshi-nishi Senior High School, Kurayoshi, Tottori, Japan.
}

state compliance $J_{\mathrm{e}}{ }^{0}$ of the blend is proportional to $w_{2}{ }^{-2}$ when $w_{2}$ is near unity, and it decreasing $w_{2}$ after passing through a maximum at around $w_{2}=0.2$. The blend of the narrow-distribution polystyrenes prepared by anionic polymerization shows the "two-step" rubbery plateau, ${ }^{4}$ indicating the existence of the two or three sets of relaxation mechanisms for entanglement slippage in the polymer blends. On the basis of these conclusions, it was suggested that Ninomiya's linear blending law should be modified into a quadratic blending law., ${ }^{4,6}$ And also, it was emphasized that the rheological studies on concentrated polymer solutions would be very important to clearify the nature of entanglement coupling between macromolecular chains. ${ }^{5,6}$

In the present paper, the viscoelastic proper- 
ties have been measured for poly(methyl methacrylate) solutions to determine the concentration dependence of the parameters such as $T_{0}$ and $\alpha_{\mathrm{f}}$, zero-shear viscosity $\eta_{0}$, steady-state compliance $J_{\mathrm{e}}{ }^{0}$, and so on.

\section{EXPERIMENTAL}

\section{Materials}

Poly(methyl methacrylate)s (PMMA) used in our previous study ${ }^{3}$ were also employed in this study. The sample were prepared by bulk polymerization and fractionated.

The weight-average molecular weight $M_{w}$, number-average molecular weight $M_{n}$ and their ratio for the PMMA samples are shown in Table I. As is evident from this Table, the

Table I. Weight- and number-average molecular weight and their ratio for poly(methyl methacrylate) samples

\begin{tabular}{cccc}
\hline Sample & $M_{w}$ & $M_{n}$ & $M_{w} / M_{n}$ \\
\hline 715 & $1.91 \times 10^{5}$ & $1.51 \times 10^{5}$ & 1.26 \\
714 & $2.29 \times 10^{5}$ & $1.75 \times 10^{5}$ & 1.31 \\
\hline
\end{tabular}

two samples have similar molecular weights and distributions. For the measurements of viscoelastic properties of the concentrated solutions, the sample 715 has been employed. And the sample 714 has been used for the measurements of zero-shear viscosity in the lower concentration range to evaluate the Vogel parameters.

Diethyl phthalate (DEP) has been used as the solvent. To prepare $40 \mathrm{wt} \%$ and more concentrated solutions, the mixture of PMMA and

Table II. Sample codes and weight fractions of poly(methyl methacrylate) solutions in diethyl phthalate(DEP) and bulk polymer

\begin{tabular}{lclc}
\hline Sample & $w_{2}$ & Sample & $w_{2}$ \\
\hline 715 & 1.000 & MC205 & 0.050 \\
MC192 & 0.920 & MC202 & 0.020 \\
MC180 & 0.800 & MC201 & 0.010 \\
MC174 & 0.740 & DEP & 0.000 \\
MC165 & 0.654 & & \\
MC150 & 0.500 & & \\
MC140 & 0.403 & & \\
MC130 & 0.300 & & \\
MC120 & 0.200 & & \\
\hline
\end{tabular}

DEP was first dissolved in benzene by stirring, and then benzene was removed by evaporation from the thin film of the solution. For preparation of solutions of lower concentration, PMMA was directly dissolved in DEP by stirring. The sample codes and the concentrations in weight fractions of polymer are tabulated in Table II. MC1 and MC2 denote the DEP solutions of 715 and 714 , respectively. The last two figures of the sample code denote the weight per cent of polymer in solution.

\section{Measurements}

The measurements of viscoelastic properties of the $\mathrm{MCl}$ series were carried out by means of the concentric cylinder type rheometer ${ }^{7}$ at various temperatures. The Maron-Krieger-Sisko visco$\operatorname{meter}^{8}$ was also used to measure the zero-shear viscosity of the MC2 series. These apparatuses are the same as those used in previous papers, ${ }^{1-5}$ and the details of the apparatuses and measuring techniques are not described here.

The viscoelastic functions, the storage shear modulus $G^{\prime}$ and loss modulus $G^{\prime \prime}$ were calculated by the usual method. The frequency dependence curves of the viscoelastic functions at various temperatures have been superposed into respective master curves according to the time-temperature superposition principle.

\section{RESULTS AND DISCUSSION}

\section{Effect of Temperature and the Vogel Parameters}

The shift factor $a_{\mathrm{T}}$ for the MC1 series and the bulk polymer 715 is plotted against temperature in Figure 1. The reference temperature $T_{\mathrm{r}}$ was chosen arbitrarily for all the samples. The plot of $-\left(T-T_{\mathrm{r}}\right) / \log a_{\mathrm{T}}$ against $\left(T-T_{\mathrm{r}}\right)$ for each sample can be represented by a straight line, and hence it is clear that the temperature dependence of the shift factor shown in this figure can be expressed by the following WLF type equation: ${ }^{9}$

$$
\log a_{\mathrm{T}}=\frac{-c_{1}^{\mathrm{r}}\left(T-T_{\mathrm{r}}\right)}{c_{2}{ }^{\mathrm{r}}+\left(T-T_{\mathrm{r}}\right)}
$$

The reference temperature $T_{\mathrm{r}}$ and two constants $c_{1}{ }^{r}$ and $c_{2}{ }^{r}$ in this equation are tabulated in Table III. It is well known ${ }^{9,10}$ that the constants in the WLF type equation can be connected with the parameters in the free volume theory by the following relations: 


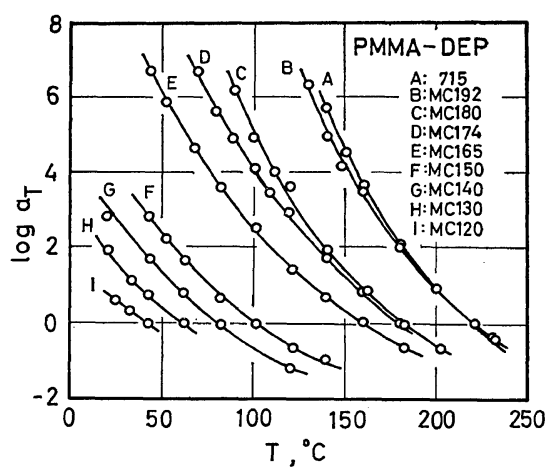

Figure 1. Temperature dependence of shift factor $a_{\mathrm{T}}$ for bulk PMMA (715) and its solutions (MC1 series).

$$
\begin{aligned}
& c_{1}{ }^{\mathrm{r}}=B / 2.303 f_{\mathrm{r}} \\
& c_{2}{ }^{\mathrm{r}}=f_{\mathrm{r}} / \alpha_{\mathrm{f}}
\end{aligned}
$$

where $B$ is the constant near unity, $f_{\mathrm{r}}$ is the fractional free volume at $T_{\mathrm{r}}$, and $\alpha_{\mathrm{f}}$ is the temperature coefficient of the fractional free volume. Equations $1-3$ are valid when the fractional free volume $f$ changes linearly with temperature difference from the constant temperature $T_{0}$, which is referred to as the Vogel temperature: ${ }^{10}$

$$
f=\alpha_{\mathrm{f}}\left(T-T_{0}\right), \quad\left(T \geq T_{\mathrm{g}}\right)
$$

The Vogel parameters $\alpha_{\mathrm{f}}$ and $T_{0}$, and $T_{\mathrm{r}}{ }^{\prime}$ calculated from eq 2, 3, and 4 are shown in Table III, where $T_{\mathrm{r}}{ }^{\prime}$ is the temperature at which each solution has the same fractional free volume, 0.062 , as that of bulk polymer 715 at $220^{\circ} \mathrm{C}$. This condition is referred to as the iso-free-volume

Table III. Reference temperature $T_{\mathrm{r}}$, the WLF constants $c_{1}{ }^{\mathrm{r}}$ and $c_{2} \mathrm{r}$, the Vogel parameters $\alpha_{\mathrm{f}}$ and $T_{0}$, and the temperature, corresponding to $f=0.062$ for bulk PMMA (715) and its solutions (MC1 series)

\begin{tabular}{lrccccc}
\hline Sample & $\begin{array}{l}T_{\mathrm{r}}, \\
{ }^{\circ} \mathrm{C}\end{array}$ & $c_{1}{ }^{\mathrm{r}}$ & $c_{2}{ }^{\mathrm{r}}$ & $\alpha_{\mathrm{f}} \times 10^{4}$ & $T_{0},{ }^{\circ} \mathrm{C}$ & $T_{\mathrm{r}}{ }^{\prime},{ }^{\circ} \mathrm{C}$ \\
\hline 715 & 220 & 7.00 & 173 & 3.59 & 47.2 & 220 \\
MC192 & 221 & 6.94 & 183 & 3.43 & 38.4 & 219 \\
MC180 & 182 & 6.99 & 181 & 3.43 & 1.0 & 182 \\
MC174 & 180 & 6.71 & 196 & 3.29 & -16.0 & 169 \\
MC165 & 160 & 6.50 & 209 & 3.20 & -48.7 & 145 \\
MC150 & 102 & 7.06 & 205 & 3.01 & -103 & 104 \\
MC140 & 83 & 6.99 & 195 & 3.19 & -112 & 82.7 \\
MC130 & 63 & 6.67 & 202 & 3.22 & -139 & 53.5 \\
MC120 & 43 & 6.25 & 205 & 3.39 & -162 & 21.0 \\
\hline
\end{tabular}

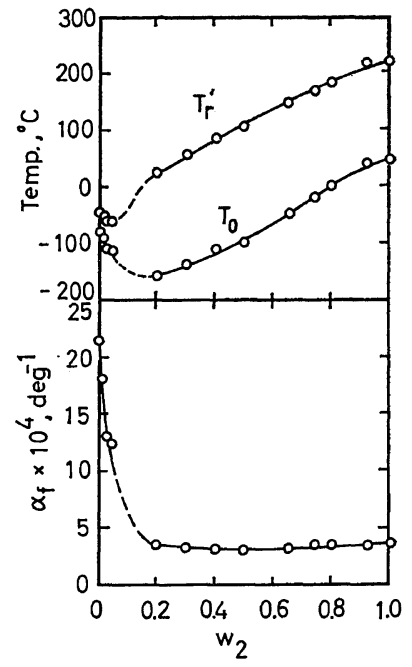

Figure 2. $T_{\mathrm{r}}{ }^{\prime}, T_{0}$ and $\alpha_{\mathrm{f}}$ plotted against weight fraction of polymer in solution for bulk PMMA (715), its solutions ( $\mathrm{MC1}$ and $\mathrm{MC} 2$ series) and the solvent DEP.

Table IV. Reference temparature $T_{\mathrm{r}}$, the WLF constans $c_{1}{ }^{\mathrm{r}}$ and $c_{2}{ }^{\mathrm{r}}$ and the Vogel parameters $\alpha_{\mathrm{f}}$ and $T_{0}$, for PMMA solutions (MC2 series) and the solvent DEP

\begin{tabular}{lccccc}
\hline Sample & $T_{\mathrm{r}},{ }^{\circ} \mathrm{C}$ & $c_{1}{ }^{\mathrm{r}}$ & $c_{2}{ }^{\mathrm{r}}$ & $\alpha_{\mathrm{f}} \times 10^{4}$ & $T_{0},{ }^{\circ} \mathrm{C}$ \\
\hline MC205 & 0 & 3.13 & 112 & 12.5 & -112 \\
MC202 & 0 & 2.98 & 111 & 13.1 & -111 \\
MC201 & 0 & 2.60 & 92.3 & 18.1 & -92.3 \\
DEP & 0 & 2.42 & 84.1 & 21.4 & -84.1 \\
\hline
\end{tabular}

state, where each polymer in solution has the same monomeric friction factor $\zeta$. Similar parameters evaluated from the temperature dependence of zero-shear viscosity are shown in Table IV for the MC2 series and the solvent, DEP. $T_{\mathrm{r}}{ }^{\prime}, T_{0}$, and $\alpha_{\mathrm{f}}$ are plotted against the weight fraction of polymer in solution, $w_{2}$, in Figure 2. With decreasing concentration, $T_{\mathrm{r}}{ }^{\prime}$ and $T_{0}$ decrease at higher concentrations and after passing through a minimum they increase at lower concentrations. A similar behavior was observed also for $T_{0}$ of binary blends of PMMA. ${ }^{5}$ The temperature coefficient of the fractional free volume, $\alpha_{\mathrm{f}}$, is almost constant at concentrations ranging from $w_{2}=1.0$ to 0.2 , as is clearly seen in Figure 2. At lower concentrations, however, $\alpha_{\mathrm{f}}$ increases rapidly with decreasing $w_{2}$. The critical concentration 
of entanglement couplings is probably located at around $w_{2}=0.2$, as will be discussed later. From the results shown in Figure 2, it can be concluded that $\alpha_{\mathrm{f}}$ of polymer solutions is almost constant in the entanglement region and changes in the lower concentration region where no continuous structure due to entanglements can be formed in the solution. This constancy of $\alpha_{\mathrm{f}}$ is also reflected in the fact that the difference between $T_{\mathrm{r}}{ }^{\prime}$ and $T_{0}$, about $190^{\circ} \mathrm{C}$, is constant, as seen from Figure 2. The fractional free volume of bulk PMMA at glass transition temperature $T_{\mathrm{g}}$ was calculated to be 0.021 , assuming $T_{\mathrm{g}}=105^{\circ} \mathrm{C}$. This value is close to the wellknown value of 0.025 but different from the literature value of 0.013 for PMMA. ${ }^{9}$

Dependence of Viscoelastic Functions on Frequency

When we compare the frequency dependence curves of viscoelastic functions of polymer solutions at various concentrations, it is important to compare them in the iso-free-valume state, where the monomeric friction factor is kept constant. In Figure 3 are shown the master

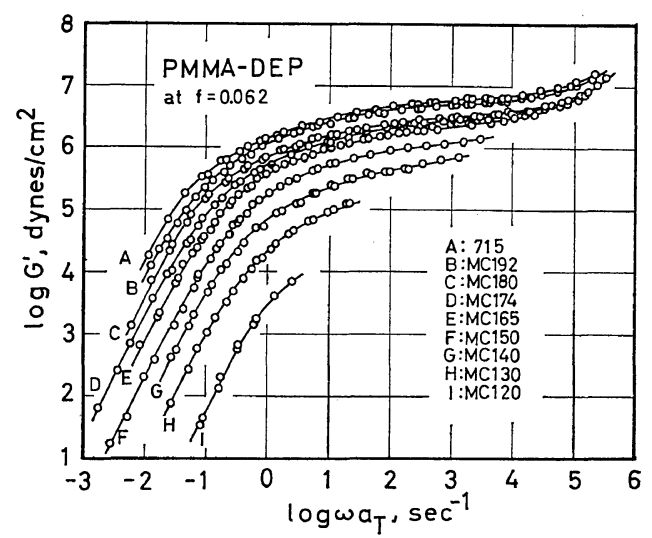

Figure 3. Master curves of $G^{\prime}$ for bulk PMMA (715) and its solutions (MC1 series) at $f=0.062$.

curves of $G^{\prime}$ for the polymer 715 and its solutions at different concentrations in the iso-freevolume state. The abscissa of the figure is the reduced angular frequency $\omega a_{T}$, and the reference temperatures are tabulated in Table III as $T_{\mathrm{r}}{ }^{\prime}$. As is evident from Figure 3, master curves for highly concentrated solutions show the rubbery plateau and terminal zone clearly. The height of the rubbery plateau becomes lower and lower, and the terminal zone shift to the higher frequency side as the concentration decreases. It is not clear whether the $G^{\prime}$ curves for solutions of low concentrations, such as MC130 and 120 , have the rubbery plateau or not. However, when we consider that the zero-shear viscosity $\eta_{0}$ is proportional to $w_{2}^{4.0}$ at $w_{2}$ between 0.2 and 1.0 in the iso-free-volume state as described below, such curves must have the rubbery plateau.

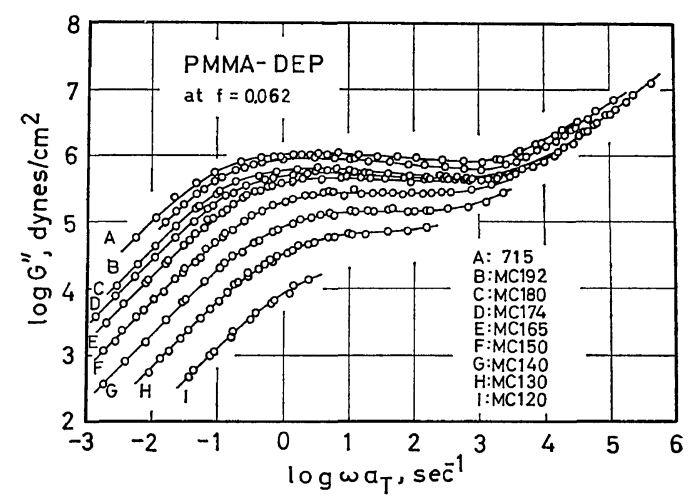

Figure 4. Master curve of $G^{\prime \prime}$ for bulk PMMA (715) and its solutions (MC1 series) at $f=0.062$.

This suggests that the critical concentration for entanglement couplings is located at about $w_{2}=$ 0.2 . The master curves for the loss modulus $G^{\prime \prime}$ at $f=0.062$ are shown in Figure 4. The frequency dependence curves of $G^{\prime \prime}$ for samples having higher concentrations show maxima in the intermediate region between the terminal and rubbery zones. Such maxima enable us to evaluate the pseudo-equilibrium modulus by the integration method proposed by Sanders, et $a .^{11}$ The results will be discussed later.

\section{Viscoelastic Properties in the Terminal Zone}

The concentration dependence of the zeroshear viscosity $\eta_{0}$ for PMMA solutions is shown in Figure 5 , where $\eta_{0}$ is defined by

$$
\eta_{0}=\lim _{\omega \rightarrow 0} G^{\prime \prime} / \omega
$$

In this figure, the closed and open circles represent, respectively, the zero-shear viscosities for the solutions at a constant temperature, $T=220^{\circ} \mathrm{C}$, and in the iso-free-volume state, where $f=0.062$. As the abscissa, the weight fraction of polymer, $w_{2}$, is employed instead of $\rho_{\mathrm{s}} w_{2}$ or volume fraction of polymer, $\phi_{2}$, where 


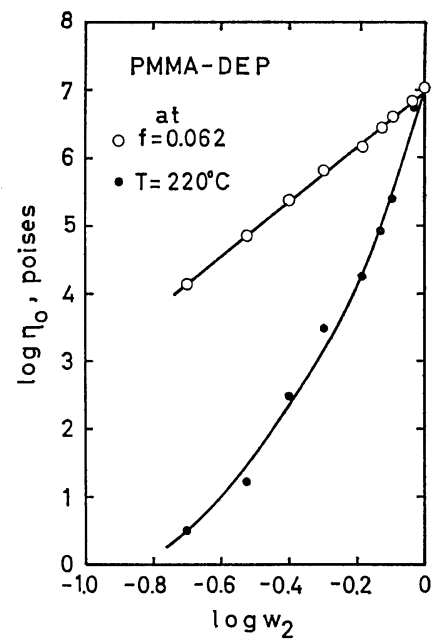

Figure 5. Concentration dependence of zero-shear viscosity $\eta_{0}$ for PMMA solutions in DEP at $220^{\circ} \mathrm{C}$ (closed circles) and $f=0.062$ (open circles).

$\rho_{\mathrm{s}}$ is the density of the solution. The densities of the solvent DEP and the solute polymer PMMA are respectively 1.12 and 1.17 at room temperature. Thermal expansion coefficient of these solutionscan be estimated to be around $5 \times 10^{-4}$ from the $\alpha_{\mathrm{f}}$ values shown in Table III and Figure 2. Therefore the density of each solution at $220^{\circ} \mathrm{C}$ is close to unity, and $w_{2}, \rho_{\mathrm{s}} w_{2}$ and $\phi_{2}$ give almost the same value when we assume the additivity of volume in mixing. Hence the use of these different expressons for concentration as the abscissa bears no serious difference in the concentration dependence curves of the visco-elastic parameters which will be discussed hereafter.

As shown in Figure 5, the concentration dependence of $\eta_{0}$ at $220^{\circ} \mathrm{C}$ (closed circles) gives a smooth curve, and its slope varies from 4 at lower concentrations to 17 at higher concentrations. The plots of $\log \eta_{0}$ against $\log w_{2}$ in the iso-free-volume state at $f=0.062$, however, can be represented by a straight line having the slope of 4.0 over the entire range of concentration. This value of the exponent of $w_{2}$ coincides well with that of the molecular weight $(M)$ dependence, $\eta_{0} \propto M^{4.0}$, found previously. ${ }^{3}$ This behovior of $\eta_{0}$ seems to support the suggestion by Fox, et al. ${ }^{10}$ that $\eta_{0}$ can be expressed by power dependence of $\left(M \phi_{2}\right): \eta_{0}$ should be proportional to $\left(M \phi_{2}\right)^{4.0}$ in this case.

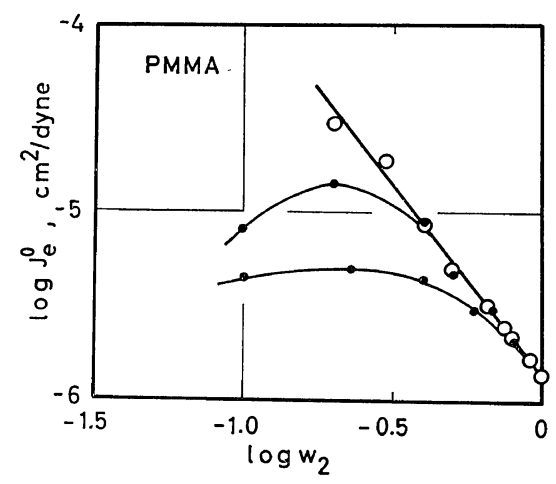

Figure 6. Concentration and composition dependence of steady-state compliance $J_{\mathrm{e}^{0}}$ for PMMA solutions in DEP (open circles) and binary blends of PMMA (closed circles).

In Figure 6 is shown the concentration dependence of the steady-state compliance $J_{\mathrm{e}}{ }^{0}$ defined by

$$
J_{\mathrm{e}}^{0}=\lim _{\omega \rightarrow 0} G^{\prime} /\left(\omega^{2} \eta_{0}^{2}\right)
$$

for the solutions (open circles) and the two binary blend series of PMMA (closed circles) reported in a previous paper. ${ }^{5}$ In the case of blends, $w_{2}$ denotes the weight fraction of the high molecular weight component having similar molecular weight and distribution to the sample 715 employed in this study. It is clear from Figure 6, that the dependence of $J_{\mathrm{e}}{ }^{0}$ on $w_{2}$ for the solutions can be represented by a straight line having the slope of -2 , which is the asymptote of the curves for the blends. This behavior of $J_{\mathrm{e}}{ }^{0}$ strongly supports our view $^{4,5}$

Table V. Numerical values of zero-shear viscosity $\eta_{0}$ at $f=0.062$, steady-state compliance $J_{\mathrm{e}}{ }^{0}$ and iso-thermal viscosity $\eta_{0}{ }^{220}$ for bulk PMMA (715) and its solutions (MC1 series)

\begin{tabular}{lccc}
\hline Sample & $\eta_{0}$, poise & $J_{\mathrm{e}}{ }^{0}, \mathrm{~cm}^{2} / \mathrm{dyn}$ & $\eta^{220}$, poise \\
\hline 715 & $1.06 \times 10^{7}$ & $1.33 \times 10^{-6}$ & $1.06 \times 10^{7}$ \\
MC192 & $6.92 \times 10^{6}$ & $1.67 \times 10^{-6}$ & $6.34 \times 10^{6}$ \\
MC180 & $4.05 \times 10^{6}$ & $2.13 \times 10^{-6}$ & $2.57 \times 10^{5}$ \\
MC174 & $2.85 \times 10^{6}$ & $2.46 \times 10^{-6}$ & $8.40 \times 10^{4}$ \\
MC165 & $1.50 \times 10^{6}$ & $3.11 \times 10^{-6}$ & $1.77 \times 10^{4}$ \\
MC150 & $6.40 \times 10^{5}$ & $4.88 \times 10^{-6}$ & $3.01 \times 10^{3}$ \\
MC140 & $2.30 \times 10^{5}$ & $8.32 \times 10^{-6}$ & $3.00 \times 10^{2}$ \\
MC130 & $7.00 \times 10^{4}$ & $1.84 \times 10^{-5}$ & $1.45 \times 10$ \\
MC120 & $1.40 \times 10^{4}$ & $2.93 \times 10^{-5}$ & $3.19 \times 10$ \\
\hline
\end{tabular}


that polymer blends and solutions should manifest similar viscoelastic behavior at long time scales. The numerical values of $\eta_{0}$ at $f=0.062$, $J_{\mathrm{e}}{ }^{0}$, and iso-thermal viscosity $\eta_{0}{ }^{220}$, at $220^{\circ} \mathrm{C}$ are tabulated in Table $\mathrm{V}$ for the bulk polymer 715 and its solutions.

\section{Viscoelastic Properties in the Rubbery Zone}

The preudo-equilibrium modulus of the entanglement network, $G_{\mathrm{eN}}^{0}$, denotes the height of the rubbery plateau in the frequency dependence curve of $G^{\prime}$. The value of $G_{\mathrm{eN}}^{0}$ can be related to the average molecular weight between entanglement coupling loci, $M_{\mathrm{e}}$, by

$$
G_{\mathrm{eN}}^{0}=g_{\mathrm{N}} \rho R T / M_{\mathrm{e}}=1 / J_{\mathrm{eN}}^{0}
$$

where $g_{N}$ is a numerical factor not far from unity and $\rho$ is the density of polymer for which $\rho_{\mathrm{s}} w_{2}$ should be used for polymer solutions, where $\rho_{\mathrm{s}}$ is the density of the solution. $J_{\mathrm{eN}}^{0}$, of reciprocal of $G_{\mathrm{eN}}^{0}$, is often referred to as the entanglement compliance. ${ }^{9}$

As seen from Figure 3, all the $G^{\prime}$ curves do not show flat rubbery plateaus, and hence it is difficult to determine the value of $G_{\mathrm{eN}}^{0}$ from the plateau accurately. In this study therefore the value of $G_{\mathrm{eN}}^{0}$ was evaluated by the following integration method proposed by Sanders, et al. $^{11}$

$$
G_{\mathrm{eN}}^{0}=\frac{2}{\pi} \int_{-\infty}^{a^{\prime}} G^{\prime \prime} \mathrm{dln} \omega
$$

The integration of $G^{\prime \prime}$ is carried out over values of $\ln \omega$ encompassing the maximum of $G^{\prime \prime} .{ }^{4}$ The calculated values of $G_{\mathrm{eN}}^{0}$ and $J_{\mathrm{eN}}^{0}$ and also of $M_{e}$ are shown in Table VI. The value of $J_{\mathrm{eN}}^{0}$ for the bulk PMMA $715,2.15 \times 10^{-7} \mathrm{~cm}^{2} /$ dyne, is consistent with the result reported in our previous paper, ${ }^{5}$ which showed that the $J_{\mathrm{eN}}^{0}$ was slightly dependent upon the molecular weight. In Figure 7, the concentration dependence of $J_{\mathrm{e} N}^{0}$ for PMMA solutions is compared with that of the steady-state compliance $J_{\mathrm{e}}{ }^{0}$. Each parameter can be represented by a straight line having the slope of -2 . This means that both $J_{\mathrm{e}}{ }^{0}$ and $J_{\mathrm{eN}}^{0}$ are proportional to $w_{2}{ }^{-2}$ and that the average molecular weight between entanglement coupling loci, $M_{\mathrm{e}}$, increases in proportion to the reciprocal of $w_{2} . \quad J_{\mathrm{e}}{ }^{0}$ is six times higher than $J_{\mathrm{eN}}^{0}$ for the PMMA solutions at and
Table VI. Pseudo-equilibrium modulus $G_{\mathrm{eN}}^{0}$, entanglement compliance $\boldsymbol{J}_{\mathrm{eN}}^{0}$ and average molecular weight between entanglement coupling loci, $M_{\mathrm{e}}$, for the bulk PMMA

(715) and its solutions (MC1 series)

\begin{tabular}{lccc}
\hline Sample & $G_{\mathrm{eN}}^{0}$, dyn $/ \mathrm{cm}^{2}$ & $J_{\mathrm{eN}}^{0}, \mathrm{~cm}^{2} / \mathrm{dyn}$ & $M_{\Theta}$ \\
\hline 715 & $4.66 \times 10^{6}$ & $2.15 \times 10^{-7}$ & $8.79 \times 10^{3}$ \\
MC192 & $4.18 \times 10^{6}$ & $2.39 \times 10^{-7}$ & $9.03 \times 10^{3}$ \\
MC180 & $2.88 \times 10^{6}$ & $3.47 \times 10^{-7}$ & $1.14 \times 10^{4}$ \\
MC174 & $2.43 \times 10^{6}$ & $4.11 \times 10^{-7}$ & $1.25 \times 10^{4}$ \\
MC165 & $2.02 \times 10^{6}$ & $4.95 \times 10^{-7}$ & $1.32 \times 10^{4}$ \\
MC150 & $1.12 \times 10^{6}$ & $8.89 \times 10^{-7}$ & $1.82 \times 10^{4}$ \\
MC140 & $6.93 \times 10^{5}$ & $1.56 \times 10^{-6}$ & $2.56 \times 10^{4}$ \\
MC130 & $3.29 \times 10^{5}$ & $3.04 \times 10^{-6}$ & $3.74 \times 10^{4}$ \\
\hline
\end{tabular}

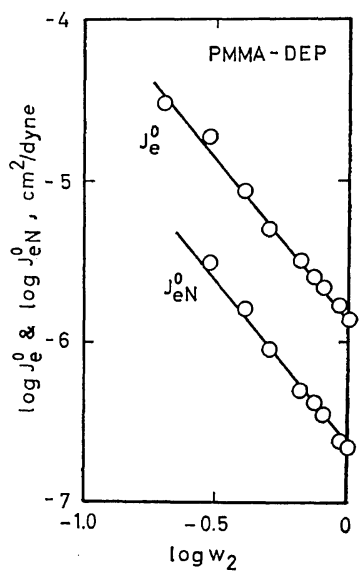

Figure 7. Concentration dependence of steadystate compliance $J_{e^{0}}$ and entanglement compliance for PMMA solutions in DEP.

above $w_{2}=0.3$.

Acknowledgement. This study was supported by a grant for scientific reseach (Kaguku Kenkyu-hi) from the Ministry of Education, Japan.

\section{REFERENCES}

1. M. Horio, T. Fujii, and S. Onogi, J. Phys. Chem., 68, 778 (1964).

2. S. Onogi, H. Kato, S. Ueki, and T. Ibaragi, J. Polym. Sci., Part C, 15, 481 (1966).

3. S. Onogi, T. Masuda, and T. Ibaragi, Kolloid Z. Z. Polym., 222, 110 (1968).

4. T. Masuda, K. Kitagawa, T. Inoue, and S. Onogi, Macromolecules, 3, 116 (1970).

5. S. Onogi, T. Masuda, N. Toda, and K. Koga, 
Viscoelastic Propertiies of Solutions of PMMA

Polymer J., 1, 542 (1970).

6. D. C. Bogue, T. Masuda, Y. Einaga, and S. Onogi, Polymer J., 1, 563 (1970).

7. M. Horio, S. Onogi, and S. Ogihara, J. Japan. Soc. Testing Mater., 10, 350 (1961).

8. S. H. Maron, I. M. Krieger, and A. W. Sisko, J. Appl. Phys., 25, 971 (1954).

9. J. D. Ferry, "Viscoelastic Properties of Poly- mers", 2nd ed, John Wiley \& Sons, Inc. N.Y., 1970.

10. G. C. Berry and T. G Fox, Advan. Polym. Sci., 5, 261 (1968).

11. J. F. Sanders, J. D. Ferry, and R. H. Valentine, J. Polym. Sci., Part A-2, 6, 967 (1968); J. F. Sanders and J. D. Ferry, Macromolecules, 2, 440 (1969). 